\title{
A Study on Fuzzy Ideals of $N$-Groups
}

\author{
B. Davvaz ${ }^{1}$ and O. Ratnabala Devi ${ }^{2}$ \\ ${ }^{1}$ Department of Mathematics, Yazd University, Yazd, Iran
}

${ }^{2}$ Department of Mathematics, Manipur University, Imphal, Manipur 795003, India

Correspondence should be addressed to B. Davvaz; davvaz@yazd.ac.ir

Received 10 February 2013; Accepted 19 June 2013

Academic Editor: Antonio M. Cegarra

Copyright (c) 2013 B. Davvaz and O. Ratnabala Devi. This is an open access article distributed under the Creative Commons Attribution License, which permits unrestricted use, distribution, and reproduction in any medium, provided the original work is properly cited.

Using the idea of the new sort of fuzzy subnear-ring of a near-ring, fuzzy subgroups, and their generalizations defined by various researchers, we try to introduce the notion of $(\epsilon, \epsilon \vee q)$-fuzzy ideals of $N$-groups. These fuzzy ideals are characterized by their level ideals, and some other related properties are investigated.

\section{Introduction and Basic Definitions}

The concept of a fuzzy set was introduced by Zadeh [1] in 1965, utilizing what Rosenfeld [2] defined as fuzzy subgroups. This was studied further in detail by different researchers in various algebraic systems. The concept of a fuzzy ideal of a ring was introduced by Liu [3]. The notion of fuzzy subnear-ring and fuzzy ideals was introduced by Abou-Zaid [4]. Then in many papers, fuzzy ideals of near-rings were discussed for example, see [5-11]. In [12], the idea of fuzzy point and its belongingness to and quasi coincidence with a fuzzy set were used to define $(\alpha, \beta)$-fuzzy subgroup, where $\alpha$, $\beta$ take one of the values from $\{\epsilon, q, \epsilon \wedge q, \epsilon \vee q\}, \alpha \neq \epsilon \wedge q$. A fuzzy subgroup in the sense of Rosenfeld is in fact an $(\epsilon, \epsilon)$-fuzzy subgroup. Thus, the concept of $(\epsilon, \epsilon \vee q)$-fuzzy subgroup was introduced and discussed thoroughly in [7]. Bhakat and Das [13] introduced the concept of $(\epsilon, \epsilon \vee q)$ fuzzy subrings and ideals of a ring. Davvaz [14, 15], Narayanan and Manikantan [16], and Zhan and Davvaz [17] studied a new sort of fuzzy subnear-ring (ideal and prime ideal) called $(\epsilon, \epsilon \vee q)$-fuzzy subnear-ring (ideal and prime ideal) and gave characterizations in terms of the level ideals. In $[18,19]$, the idea of fuzzy ideals of $\mathrm{N}$-groups was defined, and various properties such as fundamental theorem of fuzzy ideals and fuzzy congruence were studied, respectively. In the present paper, we extend the idea of $(\epsilon, \epsilon \vee q)$-fuzzy ideals of nearrings to the case of $\mathrm{N}$-groups and introduce the idea of fuzzy cosets with some results.
We first recall some basic concepts for the sake of completeness.

By a near-ring we mean a nonempty set $N$ with two binary operations "+" and "." satisfying the following axioms:

(i) $(N,+)$ is a group,

(ii) $(N, \cdot)$ is a semigroup,

(iii) $(x+y) \cdot z=x \cdot z+y \cdot z$ for all $x, y, z \in N$.

It is in fact a right near-ring because it satisfies the right distributive law. We will use the word "near-ring" to mean "right near-ring." $N$ is said to be zero symmetric if $0 \cdot x=$ $x \cdot 0=0$ for all $x \in N$. We denote $x \cdot y$ by $x y$.

Note that the missing left distributive law, $x \cdot(y+z)=$ $x \cdot y+x \cdot z$, has to do with linearity if $x$ is considered as a function.

Example 1. Let $\mathscr{G}$ be a group, and let $M(\mathscr{G})$ be the set of all mappings from $\mathscr{G}$ into $\mathscr{G}$. We define + and $\cdot$ on $M(\mathscr{G})$ by

$$
\begin{gathered}
(f+g)(x):=f(x)+g(x), \\
(f \cdot g)(x):=f(g(x)) .
\end{gathered}
$$

Then, $(M(\mathscr{G}),+, \cdot)$ is a near-ring.

Just in the same way as $R$-modules or vector spaces are used in ring theory, $N$-groups are used in near-ring theory. 
By an $N$-group we mean a nonempty set $G$ together with a map $\Phi: N \times G \rightarrow G$ written as $\Phi(n, g)=n g$ satisfying the following conditions:

(i) $(G,+)$ is a group (not necessarily abelian),

(ii) $\left(n_{1}+n_{2}\right) g=n_{1} g+n_{2} g$,

(iii) $\left(n_{1} n_{2}\right) g=n_{1}\left(n_{2} g\right)$ for all $n_{1}, n_{2} \in N, g \in G$.

Example 2. Let $N$ be a subnear-ring of $M(\mathscr{G})$. Then, $\mathscr{G}$ is an $N$-group via function application as operation.

Example 3. The additive group $(N,+)$ of a near-ring $(N,+, \cdot)$ is an $N$-group via the near-ring multiplication.

An ideal $I$ of $N$-group $G$ is an additive normal subgroup of $G$ such that $N I \subseteq I$ and $n(g+h)-n g \in I$ for all $h \in I$, $g \in G, n \in N$. A mapping between two $N$-groups $G$ and $G^{\prime}$ is called an N-homomorphism if $f(g+h)=f(g)+f(h)$ and $f(n g)=n f(g)$ for all $g, h \in G, n \in N$.

Throughout this study, we use $N$ to denote a zerosymmetric near-ring and $G$ to denote an $N$-group.

For any fuzzy subset $A$ of $G, \operatorname{Im} A=\{A(x) \mid x \in G\}$ denotes the image of $A$. For any subset $I$ of $G, \chi_{I}$ denotes the characteristic function of $I$.

Definition 4 (see [2]). A fuzzy subset $A$ of a group $G$ is called a fuzzy subgroup of $G$ if it satisfies the following conditions:

(i) $A(x+y) \geq \min \{A(x), A(y)\}$,

(ii) $A(-x) \geq A(x)$,

for all $x, y \in G$.

Definition 5. For a fuzzy subset $A$ of $G, t \in(0,1]$, the subset $A_{t}=\{x \in G \mid A(x) \geq t\}$ is called a level subset of $G$ determined by $A$ and $t$.

The set $\{x \in G \mid A(x)>0\}$ is called the support of $A$ and is denoted by $\operatorname{Supp} A$. A fuzzy subset $A$ of $G$ of the form

$$
A(y)= \begin{cases}t(\neq 0) & \text { if } y=x \\ 0 & \text { if } y \neq x\end{cases}
$$

is said to be a fuzzy point denoted by $x_{t}$. Here $x$ is called the support point, and $t$ is called its value. A fuzzy point $x_{t}$ is said to belong to (resp., quasi coincident with) a fuzzy set $A$ written as $x_{t} \in A$ (resp., $x_{t} q A$ ) if $A(x) \geq t$ (resp., $A(x)+t>1$ ). If $x_{t} \in A$ or $x_{t} q A$, then we write $x_{t} \in \vee q A$. The symbols $x_{t} \bar{\epsilon} A, x_{t} \bar{q} A, x_{t} \overline{\in \vee q A}$ mean that $x_{t} \in A, x_{t} q A, x_{t} \in \vee q A$ do not hold, respectively.

Definition 6 (see $[7,12])$. A fuzzy subset of a group $G$ is said to be an $(\epsilon, \epsilon \vee q)$-fuzzy subgroup of $G$ if for all $x, y \in G$ and $t, r \in(0,1]$,

(i) $x_{t}, y_{r} \in A \Rightarrow(x+y)_{\min \{t, r\}} \in \vee q A$,

(ii) $x_{t} \in A \Rightarrow(-x)_{t} \in \vee q A$.
Remark 7 (see [7]). The conditions (i) and (ii) of Definition 6 are respectively equivalent to

(i) $A(x+y) \geq \min \{A(x), A(y), 0.5\}$,

(ii) $A(-x) \geq \min \{A(x), 0.5\}$,

for all $x, y \in G$.

Remark 8. For any $(\epsilon, \epsilon \vee q)$-fuzzy subgroup $A$ of $G$ such that $A(x) \geq 0.5$ for some $x \in G$, then $A(0) \geq 0.5$ and if $A(0)<0.5$, then $A(x)<0.5$ for all $x \in G$. So, $A$ is just the usual fuzzy subgroup in the sense of Rosenfeld.

Remark 9. It is noted that if $A$ is a fuzzy subgroup then it is an $(\epsilon, \epsilon \vee q)$-fuzzy subgroup of $G$. However the converse may not be true.

Here onwards we assume that $A$ is an $(\epsilon, \epsilon \vee q)$-fuzzy subgroup in the nontrivial sense for which case we have $A(0) \geq 0.5$.

Definition 10 (see [7]). An ( $\epsilon, \epsilon \vee q)$-fuzzy subgroup of a group $G$ is said to be $(\epsilon, \epsilon \vee q)$-fuzzy normal subgroup if for any $x, y \in$ $G$ and $t \in(0,1]$,

$$
x_{t} \in A \Longrightarrow(y+x-y)_{t} \in \vee q A .
$$

Remark 11 (see [7]). The condition of $(\epsilon, \epsilon \vee q)$-fuzzy normal subgroup is given in the equivalent forms as

(i) $A(y+x-y) \geq \min \{A(x), 0.5\}$,

(ii) $A(x+y) \geq \min \{A(y+x), 0.5\}$,

(iii) $A([x, y]) \geq \min \{A(x), 0.5\}$, for all $x, y \in G$.

Here $[x, y]$ denotes the commutator of $x, y$ in $G$.

In the light of this fact, the condition of Definition 10 can be replaced by any one of the above conditions in Remark 8 .

Definition 12 (see [18]). Let $A$ be a fuzzy subset of an $N$ group $G$. It is called a fuzzy $N$-subgroup of $G$ if it satisfies the following conditions:

(i) $A(x+y) \geq \min \{A(x), A(y)\}$,

(ii) $A(n x) \geq A(x)$, for all $x, y \in G, n \in N$.

Remark 13. If $G$ is a unitary $N$-group, the above conditions are equivalent to conditions $A(x-y) \geq \min \{A(x), A(y)\}$ and $A(n x) \geq A(x)$ for all $x, y \in G, n \in N$.

Definition 14 (see $[18,19])$. A nonempty fuzzy subset $A$ of an $N$-group $G$ is called a fuzzy ideal if it satisfies the following conditions:

(i) $A(x-y) \geq \min \{A(x), A(y)\}$,

(ii) $A(n x) \geq A(x)$,

(iii) $A(y+x-y) \geq A(x)$,

(iv) $A(n(x+y)-n x) \geq A(y)$, for all $x, y \in G, n \in N$. 
Definition 15 (see [14]). A fuzzy set $A$ of a near-ring $N$ is called an $(\epsilon, \epsilon \vee q)$-fuzzy subnear-ring of $N$ if for all $t, r \in(0,1]$, and $x, y \in N$

(i) (a) $x_{t}, y_{r} \in A \Rightarrow(x+y)_{\min \{t, r\}} \in \vee q A$,

(b) $x_{t} \in A \Rightarrow(-x)_{t} \in \vee q A$,

(ii) $x_{t}, y_{r} \in A \Rightarrow(x y)_{\min \{t, r\}} \in \vee q A$.

$A$ is called an $(\epsilon, \epsilon \vee q)$-fuzzy ideal of $N$ if it is $(\epsilon, \epsilon \vee q)$ fuzzy subnear-ring of $N$ and

(iii) $x_{t} \in A \Rightarrow(y+x-y) \in \vee q A$,

(iv) $y_{r} \in A, x \in N \Rightarrow(y x)_{r} \in \vee q A$,

(v) $a_{t} \in A \Rightarrow(y(x+a)-y x)_{t} \in \vee q A$,

for all $x, y, a \in N$.

\section{Generalized Fuzzy Ideals}

In this section, we give the definition of $(\epsilon, \epsilon \vee q)$-fuzzy subgroup and ideal of an $N$-group $G$ based on Definitions 14 and 15.

Definition 16. A fuzzy subset $A$ of an $N$-group $G$ is said to be an $(\epsilon, \epsilon \vee q)$-fuzzy subgroup of $G$ if $x, y \in G, n \in N, t, r \in$ $(0,1]$

(i) $x_{t}, y_{r} \in A \Rightarrow(x+y)_{\min \{t, r\}} \in \vee q A$,

(ii) $x_{t} \in A \Rightarrow(-x)_{t} \in \vee q A$,

(iii) $x_{t} \in A, n \in N \Rightarrow(n x)_{t} \in \vee q A$.

Lemma 17. Let $A$ be a fuzzy subset of $G$ and $t, r \in(0,1]$. Then,

(i) $x_{t}, y_{r} \in A \Rightarrow(x+y)_{\min \{t, r\}} \in \vee q A \Leftrightarrow A(x+y) \geq$ $\min \{A(x), A(y), 0.5\}$,

(ii) $x_{t} \in A \Rightarrow(-x)_{t} \in \vee q A \Leftrightarrow A(-x) \geq \min \{A(x), 0.5\}$, for all $x \in G$,

(iii) $x_{t} \in A \Rightarrow(n x)_{t} \in \vee q A \Leftrightarrow A(n x) \geq \min \{A(x), 0.5\}$, for all $x \in G, n \in N$.

Proof. (i) Let $x, y \in G$. Consider the case (a): $\min \{A(x)$, $A(y)\}<0.5$.

Assume that $A(x+y)<\min \{A(x), A(y), 0.5\}=$ $\min \{A(x), A(y)\}$. Choose $t$ such that $A(x+y)<t<$ $\min \{A(x), A(y)\}$ which implies that $x_{t} \in A, y_{t} \in A$ but $(x+y)_{t} \overline{\epsilon \vee q A}$ [as $A(x+y)+t<1$ and $\left.A(x+y)<t\right]$. Consider the case (b): $\min \{A(x), A(y)\} \geq 0.5$. Assume that $A(x+y)<\min \{A(x), A(y), 0.5\}=0.5$. Choose $t$ such that $A(x+y)<t<0.5$ so that $x_{t}, y_{t} \in A$ but $(x+y)_{t} \overline{\in \vee q A}$.

Conversely, let $x_{t}, y_{r} \in A \Rightarrow A(x) \geq t, A(y) \geq r$. Then, $A(x+y) \geq \min \{A(x), A(y), 0.5\} \geq \min \{t, r, 0.5\}$. Thus $A(x+$ $y) \geq \min \{t, r\}$ if either $t$ or $r \leq 0.5$ and $A(x+y) \geq 0.5$ if both $t$ and $r>0.5$ which means $(x+y)_{\min \{t, r\}} \in \vee q A$.

(ii) Let $x \in G, \min \{A(x), 0.5\} \leq 0.5$. Suppose $A(-x)<$ $\min \{A(x), 0.5\} \leq 0.5$. Choose $r$ such that $A(-x)<r<$ $\min \{A(x), 0.5\} \leq 0.5$. Then, $x_{r} \in A$ but $(-x)_{r} \overline{\in \vee q A}$ which contradicts the hypothesis. So, $A(-x) \geq \min \{A(x), 0.5\}$ for all $x \in G$.
Conversely, let $x_{t} \in A$. Then, $A(x) \geq t$. But we have $A(-x) \geq \min \{A(x), 0.5\} \geq \min \{t, 0.5\} \Rightarrow A(-x) \geq t$ or $A(-x) \geq 0.5$ according as $t \leq 0.5$ or $t>0.5 \Rightarrow(-x)_{t} \in \vee q A$.

(iii) Let $x \in G$ and $\min \{A(x), 0.5\} \leq 0.5$. Suppose $A(n x)<$ $\min \{A(x), 0.5\} \leq 0.5$. Choose $r$ such that $A(n x)<r<$ $\min \{A(x), 0.5\} \leq 0.5$. Then, $A(x)>r$ that is, $x_{r} \in A$, but $(n x)_{r} \overline{\in \vee q A}$ as $A(n x)<r$ and $A(n x)+r \leq 1$.

Conversely let $x_{t} \in A, n \in N$; then $A(x) \geq t$. But $A(n x) \geq$ $\min \{A(x), 0.5\} \geq \min \{t, 0.5\} \Rightarrow A(n x) \geq t$ or $A(n x) \geq 0.5$ according as $t \leq 0.5$ or $r>0.5 \Rightarrow A(n x) \geq t$ or $A(n x)+t>$ $1 \Rightarrow(n x)_{t} \in \vee q A$.

Theorem 18. Let $A$ be a fuzzy subset of $G$. Then, $A$ is an $(\epsilon, \epsilon \vee$ q)-fuzzy subgroup of $G$ if and only if the following conditions are satisfied:

(i) $A(x+y) \geq \min \{A(x), A(y), 0.5\}$,

(ii) $A(-x) \geq \min \{A(x), 0.5\}$,

(iii) $A(n x) \geq \min \{A(x), 0.5\}$, for all $x, y \in G, n \in N$.

Proof. It follows from the previous lemma.

Definition 19. A fuzzy subset $A$ of an $N$-group $G$ is said to be $(\epsilon, \epsilon \vee q)$-fuzzy ideal of $G$ if it is an $(\epsilon, \epsilon \vee q)$-fuzzy subgroup and satisfies the following conditions:

(i) $x_{t} \in A \Rightarrow(y+x-y)_{t} \in v q A$,

(ii) $a_{t} \in A \Rightarrow(n(x+a)-n x)_{t} \in v q A$, for any $n \in N, x, a \in G$.

Lemma 20. Let $A$ be a fuzzy subset of $G$ and $t, r \in(0,1]$. Then,

(i) $x_{t} \in A \Rightarrow(y+x-y)_{t} \in v q A \Leftrightarrow A(y+x-y) \geq$ $\min \{A(x), 0.5\}$

(ii) $a_{t} \in A \Rightarrow(n(x+a)-n x)_{t} \in v q A \Leftrightarrow A(n(x+a)-n x) \geq$ $\min \{A(x), 0.5\}$.

Proof. (i) Assume that $A(y+x-y)<\min \{A(x), 0.5\}$. Choose $t$ such that $A(y+x-y)<t<\min \{A(x), 0.5\}$. But $\min \{A(x), 0.5\} \leq A(x)$ or 0.5 according as $A(x)<0.5$ or $A(x) \geq 0.5$. So, $A(x)>t$ or $A(x) \geq 0.5 \Rightarrow x_{t} \in A$ or $x_{0.5} \in A$. But $(y+x-y)_{t} \overline{\epsilon q A}$ or $(y+x-y)_{0.5} \overline{\epsilon v q A}$, respectively, which contradicts the hypothesis.

Conversely, assume that $x_{t} \in A$, then $A(x) \geq t$. For any $y \in G$, we have $A(y+x-y) \geq \min \{A(x), 0.5\} \geq \min \{t, 0.5\} \Rightarrow$ $A(y+x-y) \geq t$ or 0.5 according as $t<0.5$ or $t \geq 0.5 \Rightarrow$ $(y+x-y)_{t} \in A$ or $A(y+x-y)+t>1$. So, $\Rightarrow(y+x-y)_{t} \in v q A$.

(ii) Assume that $A(n(x+a)-n x)<\min \{A(a), 0.5\}=A(a)$ or 0.5 for some $n \in N, x, a \in G$. According as $A(a)<0.5$ or $A(a) \geq 0.5$. Choose $t \in(0,1]$ such that $A(n(x+a)-n x)<$ $t<\min \{A(a), 0.5\} \leq 0.5$. In either case, $A(n(x+a)-n x)<t$ and $A(n(x+a)-n x)+t<1$. So, $(n(x+a)-n x)_{t} \overline{\epsilon q A}$, a contradiction.

Conversely, assume that $A(n(x+a)-n x) \geq \min \{A(a), 0.5\}$ for all $a, x \in G, n \in N$. Let $a_{t} \in A$. Then, $A(a) \geq t$. So, $A(n(x+a)-n x) \geq \min \{t, 0.5\} \leq 0.5=t$ or 0.5 according as $t \leq 0.5$ or $t>0.5$. So, $A(n(x+a)-n x)_{t} \in v q A$. 
Theorem 21. Let $A$ be an $(\epsilon, \epsilon \vee q)$ fuzzy subgroup of $G$. Then, $A$ is an $(\epsilon, \epsilon \vee q)$-fuzzy ideal of $G$ if and only if

(i) $A(y+x-y) \geq \min \{A(x), 0.5\}$, for all $x, y \in G$,

(ii) $A(n(x+a)-n x) \geq \min \{A(a), 0.5\}$, for all $n \in N$, $x, a \in G$.

Proof. It is immediate from Lemma 20.

By definition, a fuzzy ideal of $G$ is an $(\epsilon, \epsilon \vee q)$-fuzzy ideal of $G$. But the converse is not true in general as shown by the following example.

Example 22. Consider $G=\mathbb{S}_{3}=\left\{i, \rho_{1}, \rho_{2}, \tau_{1}, \tau_{2}, \tau_{3}\right\}$ (written additively) to be a $\mathbb{Z}$-group. Define a fuzzy subset $A$ of $G$ as $A(i)=1, A\left(\rho_{1}\right)=A\left(\rho_{2}\right)=A\left(\tau_{2}\right)=A\left(\tau_{3}\right)=0.6$, $A\left(\tau_{1}\right)=0.8$ which is not fuzzy ideal as $A\left[2\left(\tau_{1}+\tau_{2}\right)-2 \tau_{2}\right]=$ $A\left(\rho_{1}\right)=A\left(\rho_{2}\right)=0.6<A\left(\tau_{1}\right)$; it contradicts the condition (iv) of Definition 14. As $A(x-y), A(n x), A(y+x-y)$ and $A(n(x+a)-n x)=0.6$ or $0.8 \geq \min \{0.5,0.6$ or 0.8$\}=0.5$, thus, the notion of $(\epsilon, \epsilon \vee q)$-fuzzy ideal is a successful generalization of fuzzy ideals of $G$ as introduced in [18].

Theorem 23. Let $\left\{A_{i}, i \in J\right\}$ be any family of $(\epsilon, \epsilon \vee q)$-fuzzy ideals of $G$. Then, $A=\bigcap A_{i}$ is an $(\epsilon, \epsilon \vee q)$-fuzzy ideal of $G$.

Proof. It is straightforward.

Theorem 24. A nonempty subset I of $G$ is an ideal of $G$ if and only if $\chi_{I}$ is an $(\epsilon, \in \vee q)$-fuzzy ideal of $G$.

Proof. If $I$ is an ideal of $G$, it is clear from [18, Proposition 2.11] that $\chi_{I}$ is fuzzy ideal of $G$. Since every fuzzy ideal is $(\epsilon, \epsilon \vee q)$ fuzzy ideal, $\chi_{I}$ is $(\epsilon, \epsilon \vee q)$-fuzzy ideal of $G$.

Conversely, let $\chi_{I}$ be an $(\epsilon, \epsilon \vee q)$-fuzzy ideal of $G$. Let $x, y \in I, \chi_{I}(x-y) \geq \min \left\{\chi_{I}(x), \chi_{I}(y), 0.5\right\}=0.5$. So, $\chi_{I}(x-y)=1 \Rightarrow x-y \in I$. Let $n \in N, x \in I, \chi_{I}(n x) \geq$ $\min \left\{\chi_{I}(x), 0.5\right\}=0.5 \Rightarrow \chi_{I}(n x)=1 \Rightarrow n x \in I y \in G, x \in I$, $\chi_{I}(y+x-y) \geq \min \left\{\chi_{I}(x), 0.5\right\}=0.5 \Rightarrow \chi_{I}(y+x-y)=$ $1 \Rightarrow y+x-y \in I n \in N, x \in I, y \in G, \chi_{I}(n(y+x)-n y) \geq$ $\min \left\{\chi_{I}(x), 0.5\right\}=0.5 \Rightarrow \chi_{I}(n(y+x)-n y)=1 \Rightarrow n(y+x)-$ $n y \in I$. Then, $I$ is an ideal of $G$.

Theorem 25. A fuzzy subset $A$ of $G$ is an ( $\epsilon, \epsilon \vee q)$-fuzzy (subgroup) ideal of $G$ if and only if the level subset $A_{t}$ is a (subgroup) ideal for $0<t \leq 0.5$.

Proof. We prove the result for $(\epsilon, \epsilon \vee q)$-fuzzy ideal $S$. Let $A$ be an $(\epsilon, \epsilon \vee q)$-fuzzy ideal of $G$. Let $t \leq 0.5, x, y, i \in A_{t}, n \in N$.

(i) $A(x-y) \geq \min \{A(x), A(y), 0.5\} \geq \min \{t, 0.5\}=t \Rightarrow$ $x-y \in A_{t}$,

(ii) $A(n x) \geq \min \{A(a), 0.5\} \geq \min \{t, 0.5\}=t \Rightarrow n x \in$ $A_{t}$,

(iii) $A(y+x-y) \geq \min \{A(x), 0.5\} \geq \min \{t, 0.5\}=t \Rightarrow$ $y+x-y \in A_{t}, y \in G$

(iv) $A(n(y+x)-n y) \geq \min \{A(x), 0.5\} \geq \min \{t, 0.5\}=$ $t \Rightarrow n(y+x)-n y \in A_{t}, y \in G, n \in N$.
Hence, $A_{t}$ is an ideal of $G$. Again, let $A_{t}$ be an ideal of $G$ for all $t \leq 0.5$. If possible, let there exist $x, y \in G$ such that $A(x-$ $y)<t<\min \{A(x), A(y), 0.5\}$. Let $t$ be such that $A(x-y)<$ $t<\min \{A(x), A(y), 0.5\} \Rightarrow x, y \in A_{t}$ and $x-y \notin A_{t}$, a contradiction. So, $A(x-y) \geq \min \{A(x), A(y), 0.5\}$, for all $x, y \in G$. For $n \in N, x \in G$ let $A(n x)<\min \{A(x), 0.5\}$. If possible let $t$ be such that $A(n x)<t<\min \{A(x), 0.5\}$. This implies $x \in A_{t}$, but $n x \notin A_{t}$, a contradiction. Similarly, we can prove that $A(y+x-y) \geq \min \{A(x), 0.5\}, A[n(y+x)-$ $n y] \geq \min \{A(x), 0.5\}, x, y \in G, n \in N$.

Remark 26. For $t \in(0.5,1), A$ may be an $(\epsilon, \epsilon \vee q)$-fuzzy ideal of $G$, but $A_{t}$ may not be an ideal of $G$. Let $t=0.8$ in Example 22. Then, $A_{t}=\left\{i, \tau_{1}\right\}$. $A_{t}$ is not an ideal of $\mathbb{S}_{3}$ as it is not a normal subgroup of $\mathbb{S}_{3}$.

We are looking for a corresponding result when $A_{t}$ is an ideal of $G$ for all $t \in(0.5,1]$.

Theorem 27. Let $A$ be a fuzzy subset of an $N$-group G. Then, $A_{t} \neq \phi$ is an ideal of $G$ for all $t \in(0.5,1]$ if and only if $A$ satisfies the following conditions:

(i) $\max \{A(x-y), 0.5\} \geq \min \{A(x), A(y)\}$,

(ii) $\max \{A(n x), 0.5\} \geq A(x)$,

(iii) $\max \{A(y+x-y), 0.5\} \geq A(x)$,

(iv) $\max \{A(n(y+x)-n y), 0.5\} \geq A(x)$,

for all $x, y \in G, n \in N$.

Proof. Suppose that $A_{t} \neq \phi$ is an ideal of $G$ for all $t \in$ $(0.5,1]$. In order to prove (i), suppose that for some $x, y \in$ $G, \max \{A(x-y), 0.5\}<\min \{A(x), A(y)\}$. Let $t=$ $\min \{A(x), A(y)\}$. So, $x, y \in A_{t}$ and $t \in(0.5,1]$. Since $A_{t}$ is an ideal, $x-y \in A_{t}$. So, $A(x-y) \geq t>\max \{A(x-y), 0.5\}$, a contradiction. In order to prove (ii), suppose that $x \in G$, $n \in N$ and $\max \{A(n x), 0.5\}<A(x)=t$ (say). Then, $x \in A_{t} \Rightarrow n x \in A_{t} \Rightarrow A(n x) \geq t>\max \{A(n x), 0.5\}$, a contradiction. Similarly, we can prove (iii) and (iv).

Conversely, suppose that conditions (i) to (iv) hold. We show that $A_{t}$ is an ideal of $G$ for all $t \in(0.5,1]$. Let $x, y \in A_{t}$. Then, $0.5<t \leq \min \{A(x), A(y)\} \leq \max \{A(x-y), 0.5\}=$ $A(x-y)$. So, $x-y \in A_{t}$. Let $n \in N, x \in A_{t}$. Then, $0.5<$ $t \leq A(x) \leq \max \{A(x), 0.5\}=A(x)$ so $n x \in A_{t}$. For $x \in A_{t}$, $y \in G, 0.5<t \leq A(x) \leq \max \{A(y+x-y), 0.5\}=A(y+x-$ $y) \Rightarrow y+x-y \in A_{t}$. Also, if $n \in N, x \in A_{t}, y \in G, 0.5<$ $t \leq A(x) \leq \max \{A(n(y+x)-n y), 0.5\}=A(n(y+x)-n y)$. Hence, $n(y+x)-n y \in A_{t}$. Then, $A_{t}$ is an ideal of $G$.

A definition for the previous kind of fuzzy subset was given for the case of near-rings in [17]. Now, we give the definition for $N$-groups.

Definition 28. A fuzzy subset of $G$ is called an $(\bar{\epsilon}, \bar{\epsilon} \vee \bar{q})-f u z z y$ subgroup of $G$ if for all $t, r \in(0,1]$ and for all $x, y \in G, n \in N$,

(i) (a) $(x+y)_{\min (t, r)} \bar{\epsilon} A$ implies $x_{t} \bar{\epsilon} v \bar{q} A$ or $y_{r} \bar{\epsilon} v \bar{q} A$,

(b) $(-x)_{t} \bar{\epsilon} A$ implies $x_{t} \bar{\epsilon} v \bar{q} A$, 
(ii) $(n x)_{t} \bar{\epsilon} A$ implies $x_{t} \bar{\epsilon} v \bar{q} A$.

Moreover, $A$ is called an $(\bar{\epsilon}, \bar{\epsilon} \vee \bar{q})$-fuzzy ideal of $G$ if $A$ is $(\bar{\epsilon}, \bar{\epsilon} \vee \bar{q})$-fuzzy subgroup of $G$ and

(iii) $(y+x-y)_{t} \bar{\epsilon} A$ implies $x_{t} \bar{\epsilon} v \bar{q} A$,

(iv) $(n(x+y)-n y)_{t} \bar{\epsilon} A$ implies $x_{t} \bar{\epsilon} v \bar{q} A$.

Theorem 29. A fuzzy subset $A$ of $G$ is an $(\bar{\epsilon}, \bar{\epsilon} \vee \bar{q})$-fuzzy ideal of $G$ if and only if
(1) (a) $\max \{A(x+y), 0.5\} \geq \min \{A(x), A(y)\}$,
(b) $\max \{A(-x), 0.5\} \geq A(x)$,
(2) $\max \{A(y+x-y), 0.5\} \geq A(x)$,
(3) $\max \{A(n x), 0.5\} \geq A(x)$,
(4) $\max \{A(n(y+x)-n y), 0.5\} \geq A(x)$.

Proof. (i)a $\Leftrightarrow$ (1)a. Let $x, y \in G$ be such that $\max \{A(x+$ $y), 0.5\}<\min \{A(x), A(y)\}$. Let $t=\min \{A(x), A(y)\}$; then $0.5<t \leq 1,(x+y)_{t} \bar{\epsilon} A$. So we must have $x_{t} \bar{\epsilon} v \bar{q} A$ or $y_{t} \bar{\epsilon} v \bar{q} A$. But $x_{t} \in A$ and $y_{t} \in A$. Here $x_{t} \bar{q} A$ or $y_{t} \bar{q} A$ then $t \leq A(x)$ and $A(x)+t \leq 1$ or $t \leq A(y)$ and $A(y)+t \leq 1$ then $t \leq 0.5$, a contradiction.

Conversely, let $(x+y)_{\min (t, r)} \bar{\epsilon} A$. Then, $A(x+y)<\min (t$, $r)$. If $A(x+y) \geq \min \{A(x), A(y)\}$, then $\min \{A(x), A(y)\}<$ $\min (t, r)$. Hence, either $A(x)<t$ or $A(y)<r$ which implies $x_{t} \bar{\epsilon} A$ or $y_{r} \bar{\epsilon} A$. Thus, $x_{t} \bar{\epsilon} v \bar{q} A$ or $y_{r} \bar{\epsilon} v \bar{q} A$.

Again if $A(x+y)<\min \{A(x), A(y)\}$, then by (1)a

$$
0.5 \geq \min \{A(x), A(y)\}>A(x+y) .
$$

Suppose that $x_{t} \in A$ or $y_{r} \in A$ then $t \leq A(x) \leq 0.5$ or $r \leq A(y) \leq 0.5$. It follows that either $x_{t} \bar{q} A$ or $y_{t} \bar{q} A$, and thus $x_{t} \bar{\epsilon} v \bar{q} A$ or $y_{r} \bar{\epsilon} v \bar{q} A$.

(i) $\mathrm{b} \Leftrightarrow$ (1)b: Suppose that there exists $x \in G$ such that $\max \{A(-x), 0.5\}<A(x)$. If $A(x)=t$ then $0.5<t \leq 1$ and $A(-x)<t$ so that $(-x)_{t} \bar{\epsilon} A$. But then we must have either $(x)_{t} \bar{\epsilon} A$ or $x_{t} \bar{q} A$. Also we have $(x)_{t} \in A$. So, $A(x)+1 \leq 1$ which means that $t \leq 0.5$, a contradiction.

Conversely, suppose that $(x)_{t} \bar{\epsilon} A$ then $A(-x)<t$. If $A(-x) \geq A(x)$, then $A(x)<t$ which gives $x_{t} \bar{\epsilon} v \bar{q} A$. Again if $A(-x)<A(x)$ by (1)b we have $0.5 \geq A(x)$. Putting $(x)_{t} \in A$, then $t \leq A(x) \leq 0.5$ so that $x_{t} \bar{q} A$ which means that $x_{t} \bar{\epsilon} v \bar{q} A$. Similarly, we can prove the remaining parts.

Theorem 30. A fuzzy subset $A$ of $G$ is an $(\bar{\epsilon}, \bar{\epsilon} v \bar{q})$-fuzzy ideal if and only if $A_{t}(\neq \phi)$ is an ideal of $G$ for all $t \in(0.5,1]$.

\section{Fuzzy Cosets and Isomorphism Theorem}

In this section, we first study the properties of $(\epsilon, \epsilon \vee q)$-fuzzy ideals under a homomorphism. Then, we introduce the fuzzy cosets and prove the fundamental isomorphism theorem on $N$-groups with respect to the structure induced by these fuzzy cosets.

Theorem 31. Let $G$ and $G^{\prime}$ be two N-groups, and let $f: G \rightarrow$ $G^{\prime}$ be an $N$-homomorphism. If $f$ is surjective and $A$ is an $(\epsilon, \epsilon \vee$ q)-fuzzy ideal of $G$, then so is $f(A)$. If $B$ is a $(\epsilon, \epsilon \vee q)$-fuzzy ideal of $G^{\prime}$, then $f^{-1}(B)$ is a fuzzy ideal of $G$.
Proof. We assume that $A$ is an $(\epsilon, \epsilon \vee q)$-fuzzy ideal of $G$. For any $x, y \in G^{\prime}$; it follows that

$$
\begin{aligned}
f(A)(x+y) & =\sup _{x+y=f(z)}\{A(z)\} \\
\geq & \sup _{f(u)=x, f(v)=y}\{A(u+v)\} \\
\geq & \sup _{f(u)=x, f(v)=y}\{\min \{A(u), A(v), 0.5\}\} \\
= & \min \left\{\sup _{f(u)=x}\{A(u)\}, \sup _{f(v)=y}\{A(v)\}, 0.5\right\} \\
& =\min \{f(A)(x), f(A)(y), 0.5\} .
\end{aligned}
$$

Also,

$$
\begin{aligned}
f(A) & (-x) \\
= & \sup _{f(z)=-x}\{A(z)\}=\sup _{f(-z)=x}\{A(z)\} \\
= & \sup _{f(z)=x}\{A(-z)\} \\
\geq & \sup _{f(z)=x}\{\min \{A(u), 0.5\}\} \\
= & \min \left\{\sup _{f(z)=x}\{A(z)\}, 0.5\right\} \\
= & \min \{f(A)(x), 0.5\} .
\end{aligned}
$$

Again,

$$
\begin{aligned}
& f(A)(n x) \\
&= \sup _{f(z)=n x}\{A(z)\} \geq \sup _{f(u)=x}\{A(n u)\} \\
& \geq \sup _{f(u)=x}\{\min \{A(u), 0.5\}\} \\
&= \min \left\{\sup _{f(u)=x}\{A(u)\}, 0.5\right\} \\
&= \min \{f(A)(x), 0.5\}, \\
& f(A)(y+x-y) \\
&=\sup _{f(z)=y+x-y}\{A(z)\} \\
& \geq \sup _{f(v)=x, f(u)=y}\{A(u+v-u)\}
\end{aligned}
$$




$$
\begin{aligned}
& \geq \sup _{f(v)=x}\{\min \{A(v), 0.5\}\} \\
& =\min \left\{\sup _{f(v)=x}\{A(v)\}, 0.5\right\} \\
& =\min \{f(A)(x), 0.5\}, \\
& f(A)(n(y+x)-n y) \\
& =\sup _{f(z)=n(y+x)-n y}\{A(z)\} \\
& \geq \sup _{f(v)=x, f(u)=y}\{A(n(u+v)-n u)\} \\
& \geq \sup _{f(v)=x, f(u)=y}\{\min \{A(u), 0.5\}\} \\
& =\min \{f(A)(x), 0.5\} .
\end{aligned}
$$

Therefore, $f(A)$ is an $(\epsilon, \epsilon \vee q)$-fuzzy ideal of $G$. Similarly, we can show that $f^{-1}(A)$ is an $(\epsilon, \epsilon \vee q)$-fuzzy ideal of $G$.

Definition 32. Let $A$ be $(\epsilon, \in \vee q)$-fuzzy subgroup of $G$. For any $x \in G$, let $A_{x}$ be defined by $A_{x}(g)=\min \{A(g-x), 0.5\}$ for all $g \in G$. This fuzzy subset $A_{x}$ is called the $(\epsilon, \in \vee q)$-fuzzy left coset of $G$ determined by $A$ and $x$.

Remark 33. Let $A$ be an $(\epsilon, \epsilon \vee q)$-fuzzy subgroup of $G$. Then, $A$ is an $(\epsilon, \epsilon \vee q)$-fuzzy normal if and only if $\min \{A(g-$ $x), 0.5\}=\min \{A(-x+g), 0.5\}$ for all $x, g \in G$. If $A$ is an $(\epsilon, \epsilon \vee q)$-fuzzy ideal, we simply denote fuzzy coset by $A_{x}$.

Lemma 34. Let $A$ be an $(\epsilon, \epsilon \vee q)$-fuzzy ideal of $G$. Then, $A_{x}=A_{y}$ if an only if $A(x-y) \geq 0.5$.

Proof. Assume that $A(x-y) \geq 0.5$ and $g \in G$. $A_{x}(g)=$ $\min \{A(g-x), 0.5\} \geq \min \{A(g-y), A(y-x), 0.5\} \geq \min \{A(g-$ $y), A(x-y), 0.5\}=\min \{A(g-y), 0.5\}=A_{y}(g)$ which implies that $A_{x} \geq A_{y}$. Similarly, we can verify that $A_{x} \leq$ $A_{y}$. Conversely we assume that $A_{x}=A_{y}$. Then, $A_{y}(x)=$ $A_{x}(x) \Rightarrow \min \{A(x-y), 0.5\}=0.5 \Rightarrow A(x-y) \geq 0.5$.

Proposition 35. Every fuzzy coset $A_{x}$ is constant on every coset of $G_{0}=\{x \in G \mid A(x)=A(0)\}$.

Proof. Let $y+y_{0} \in y+G_{0}$. Now, we have $A_{x}\left(y+y_{0}\right)=$ $\min \left\{A\left(y+y_{0}-x\right), 0.5\right\} \geq \min \left\{A(y-x), A\left(x+y_{0}-x\right), 0.5\right\} \geq$ $\min \left\{A(y-x), A\left(y_{0}\right), 0.5\right\}=\min \{A(y-x), 0.5\}=A_{x}(y)$. Also $A_{x}(y)=\min \{A(y-x), 0.5\} \geq \min \left\{A\left(-x+y+y_{0}-y_{0}\right), 0.5\right\} \geq$ $\min \left\{A\left(-x+y+y_{0}\right), A\left(-y_{0}\right), 0.5\right\}=\min \left\{A\left(-x+y+y_{0}\right), 0.5\right\} \geq$ $\min \left\{A\left(y+y_{0}-x\right), 0.5\right\}=A_{x}\left(y+y_{0}\right)$. Thus $A_{x}\left(y+y_{0}\right)=A_{x}(y)$ for all $y_{0} \in G_{0}$.

Theorem 36. For any $(\epsilon, \epsilon \vee q)$-fuzzy ideal $A$ of $G, G_{A}$ the set of all fuzzy cosets of $A$ in $G$ is an $N$-group under the addition and scalar multiplication defined by $A_{x}+A_{y}=A_{x+y}, n\left(A_{x}\right)=$ $A_{n x}$ for all $x, y \in G, n \in N$. The function $\bar{A}\left(A_{x}\right): G_{A} \rightarrow$
$[0,1]$ defined by $\bar{A}\left(A_{x}\right)=A(x)$ for all $A_{x} \in G_{A}$ is $(\epsilon, \epsilon \vee q)$ fuzzy ideal of $G_{A}$.

Proof. First, we show that the compositions are well defined. Let $x, y, c, d \in G, n \in N$ such that $A_{x}=A_{y}$ and $A_{c}=A_{d}$. Then, $A(x-y) \geq 0.5, A(c-d) \geq 0.5$. Now, we have $\min \{A(x+$ $c-d-y), 0.5\} \geq \min \{A(-y+x+c-d), 0.5\}=0.5$ which implies that $A(x+c-d-y) \geq 0.5$. So, by Lemma 34, we have $A_{x+c}=$ $A_{y+d}$. Again $A_{n x}(g)=\min \{A(g-n x), 0.5\} \geq \min \{A(g-$ $n y), A(n x-n y), 0.5\}=\min \{A(g-n y), A[n(y-y+x)-$ $n y], 0.5\} \geq \min \{A(g-n y), A(-y+x), 0.5\}=A_{n y}(g)$. Similarly, we show that $A_{n y}(g) \geq A_{n x}(g)$. Thus, for $A_{n y}(g)=A_{n x}(g)$ for all $g \in G$. Hence the compositions are well defined. It is now easy to verify that $G_{A}$ is an $N$-group with null element $A_{0}$ and negative element $A_{-x}$. Next, we check that $\bar{A}$ is $(\epsilon, \epsilon \vee q)$ fuzzy ideal of $G_{A}$. Let $A_{x}, A_{y} \in G_{A}$. We have

(i) $\bar{A}\left(A_{x}-A_{y}\right)=\bar{A}\left(A_{x-y}\right)=A(x-y) \geq \min \{A(x), A(y)$, $0.5\}=\min \left\{\bar{A}\left(A_{x}\right), \bar{A}\left(A_{y}\right), 0.5\right\}$,

(ii) $\bar{A}\left(A_{n x}\right)=A(n x) \geq \min \{A(x), 0.5\}=\min \left\{\bar{A}\left(A_{x}\right)\right.$, $0.5\}$

(iii) $\bar{A}\left(A_{y}+A_{x}-A y\right)=\bar{A}\left(A_{y+x-y}\right)=A(y+x-y) \geq$ $\min \{A(x), 0.5\}=\min \left\{\bar{A}\left(A_{x}\right), 0.5\right\}$,

(iv) $\bar{A}\left[n\left(A_{y}+A_{x}\right)-n A_{y}\right]=\bar{A}\left[A_{n(y+x)-n y}\right]=A[n(y+x)-$ $n y] \geq \min \{A(x), 0.5\}=\min \left\{\bar{A}\left(A_{x}\right), 0.5\right\}$.

Hence, the proof is completed.

Lemma 37. Let $H=\left\{x \in G \mid A_{x} \geq A_{0}\right\}, K=\left\{x \in G \mid A_{x}=\right.$ $\left.A_{0}\right\}$. Then, $K=H=A_{0.5}$.

Proof. Let $x \in H$. Then, for all $g \in G, A_{x}(g) \geq A_{0}(g) \Rightarrow$ $\min \{A(g-x), 0.5\} \geq \min \{A(g), 0.5\} \Rightarrow \min \{A(-x), 0.5\} \geq$ $\min \{A(0), 0.5\}=0.5 \Rightarrow A(x) \geq 0.5$. Also, for $g \in G$, $A_{0}(g)=\min \{A(g), 0.5\}=\min \{A(g-x+x), 0.5\} \geq$ $\min \{A(g-x), A(x), 0.5\}=\min \{A(g-x), 0.5\}=A_{x}(g) \Rightarrow$ $A_{0} \geq A_{x}$. So, $H=K$. As we have seen, if $x \in H$ then $A(x) \geq 0.5 \Rightarrow x \in A_{0.5}$. Conversely, if $x \in A_{0.5}$, for any $g \in G, A(g-x) \geq \min \{A(g), A(x), 0.5\}=\min \{A(g), 0.5\} \Rightarrow$ $\min \{A(g-x), 0.5\} \geq \min \{A(g), 0.5\} \Rightarrow A_{x} \geq A_{0}$. Thus, $H=A_{0.5}$, which means that $H$ is an ideal of $G$.

Theorem 38. If $A$ is an $(\epsilon, \epsilon \vee q)$-fuzzy ideal of $G$, then the map $f: G \rightarrow G_{A}$ given by $f(x)=A_{x}$ is an N-homomorphism with kernel $f=A_{0.5}$ and so $G / A_{0.5}$ is isomorphic to $G_{A}$.

Proof. It is clear that $f$ is an onto $N$-homomorphism from $G$ to $G_{A}$ with kernel $f=\{x \in G \mid f(x)=f(0)\}=\{x \in G \mid$ $\left.A_{x}=A_{0}\right\}=A_{0.5}$.

Corollary 39. Let $A$ be an $(\epsilon, \epsilon \vee q)$-fuzzy ideal of $G$, and let $\bar{B}$ be an $(\epsilon, \epsilon \vee q)$-fuzzy ideal of $G_{A}$. Then, $B: G \rightarrow[0,1]$ defined by $B(x)=\bar{B}\left(A_{x}\right)$ is an $(\epsilon, \epsilon \vee q)$-fuzzy ideal of $G$ containing $A$.

\section{References}

[1] L. A. Zadeh, "Fuzzy sets," Information and Control, vol. 8, no. 3, pp. 338-353, 1965. 
[2] A. Rosenfeld, "Fuzzy groups," Journal of Mathematical Analysis and Applications, vol. 35, pp. 512-517, 1971.

[3] W. J. Liu, "Fuzzy invariant subgroups and fuzzy ideals," Fuzzy Sets and Systems, vol. 8, no. 2, pp. 133-139, 1982.

[4] S. Abou-Zaid, "On fuzzy subnear-rings and ideals," Fuzzy Sets and Systems, vol. 44, no. 1, pp. 139-146, 1991.

[5] B. Davvaz, "Fuzzy ideals of near-rings with interval valued membership functions," Journal of Sciences, Islamic Republic of Iran, vol. 12, no. 2, pp. 171-175, 2001.

[6] K. H. Kim, "On vague $R$-subgroups of near-rings," International Mathematical Forum, vol. 4, no. 33-36, pp. 1655-1661, 2009.

[7] S. K. Bhakat and P. Das, “( $\epsilon, \epsilon \vee q)$-fuzzy subgroup," Fuzzy Sets and Systems, vol. 80, no. 3, pp. 359-368, 1996.

[8] K. H. Kim, Y. B. Jun, and Y. H. Yon, "On anti fuzzy ideals in near-rings," Iranian Journal of Fuzzy Systems, vol. 2, no. 2, pp. 71-80, 2005.

[9] K. H. Kim and Y. B. Jun, "A note on fuzzy $R$-subgroups of nearrings," Soochow Journal of Mathematics, vol. 28, no. 4, pp. 339346, 2002.

[10] S. D. Kim and H. S. Kim, "On fuzzy ideals of near-rings," Bulletin of the Korean Mathematical Society, vol. 33, no. 4, pp. 593-601, 1996.

[11] O. R. Devi, "Intuitionistic fuzzy near-rings and its properties," The Journal of the Indian Mathematical Society, vol. 74, no. 3-4, pp. 203-219, 2007.

[12] S. K. Bhakat and P. Das, "On the definition of a fuzzy subgroup," Fuzzy Sets and Systems, vol. 51, no. 2, pp. 235-241, 1992.

[13] S. K. Bhakat and P. Das, "Fuzzy subrings and ideals redefined," Fuzzy Sets and Systems, vol. 81, no. 3, pp. 383-393, 1996.

[14] B. Davvaz, "( $\epsilon, \epsilon \vee q)$-fuzzy subnear-rings and ideals," Soft Computing, vol. 10, no. 3, pp. 206-211, 2006.

[15] B. Davvaz, "Fuzzy $R$-subgroups with thresholds of near-rings and implication operators," Soft Computing, vol. 12, no. 9, pp. 875-879, 2008.

[16] A. Narayanan and T. Manikantan, “( $\epsilon, \epsilon \vee q)$-fuzzy subnearrings and $(\epsilon, \epsilon \vee q)$-fuzzy ideals of near-rings," Journal of Applied Mathematics \& Computing, vol. 18, no. 1-2, pp. 419-430, 2005.

[17] J. Zhan and B. Davvaz, "Generalized fuzzy ideals of near-rings," Applied Mathematics, vol. 24, no. 3, pp. 343-349, 2009.

[18] O. R. Devi and M. R. Singh, "On fuzzy ideals of N-groups," Bulletin of Pure \& Applied Sciences E, vol. 26, no. 1, pp. 11-23, 2007.

[19] T. K. Dutta and B. K. Biswas, "On fuzzy congruence of a nearring module," Fuzzy Sets and Systems, vol. 112, no. 2, pp. 343348, 2000. 


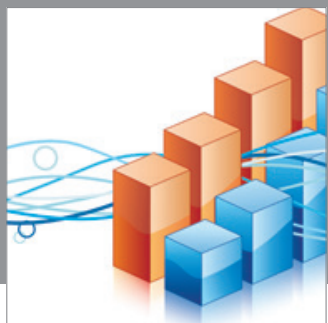

Advances in

Operations Research

mansans

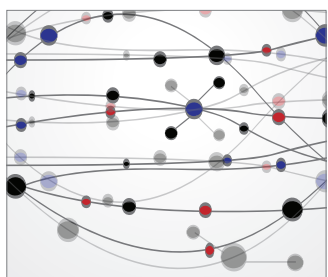

The Scientific World Journal
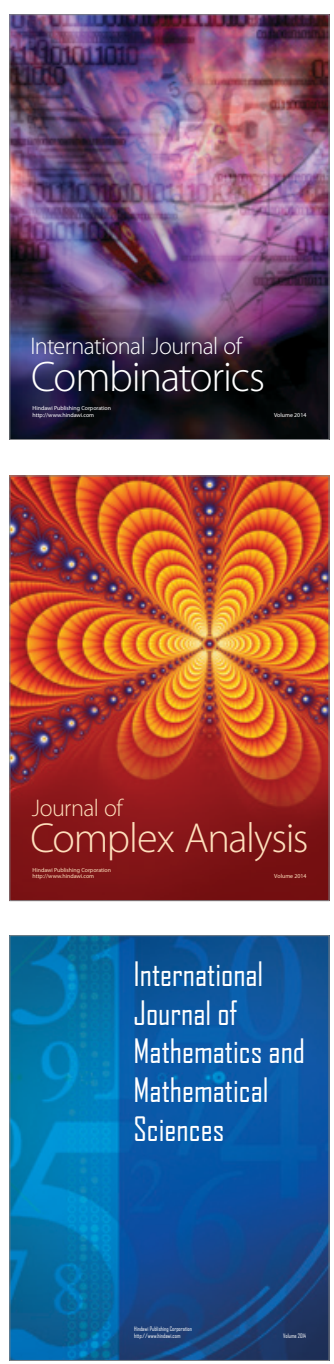
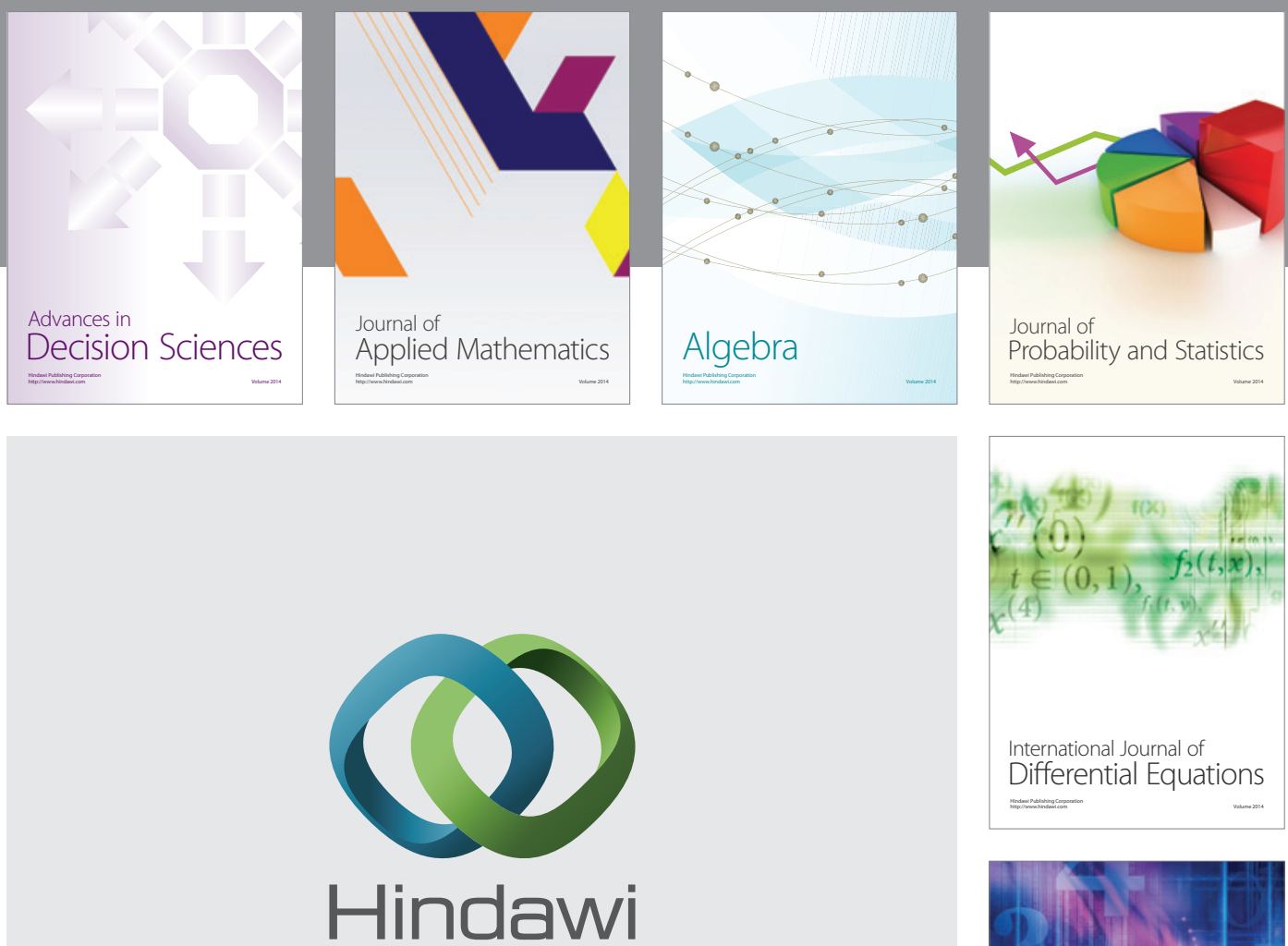

Submit your manuscripts at http://www.hindawi.com
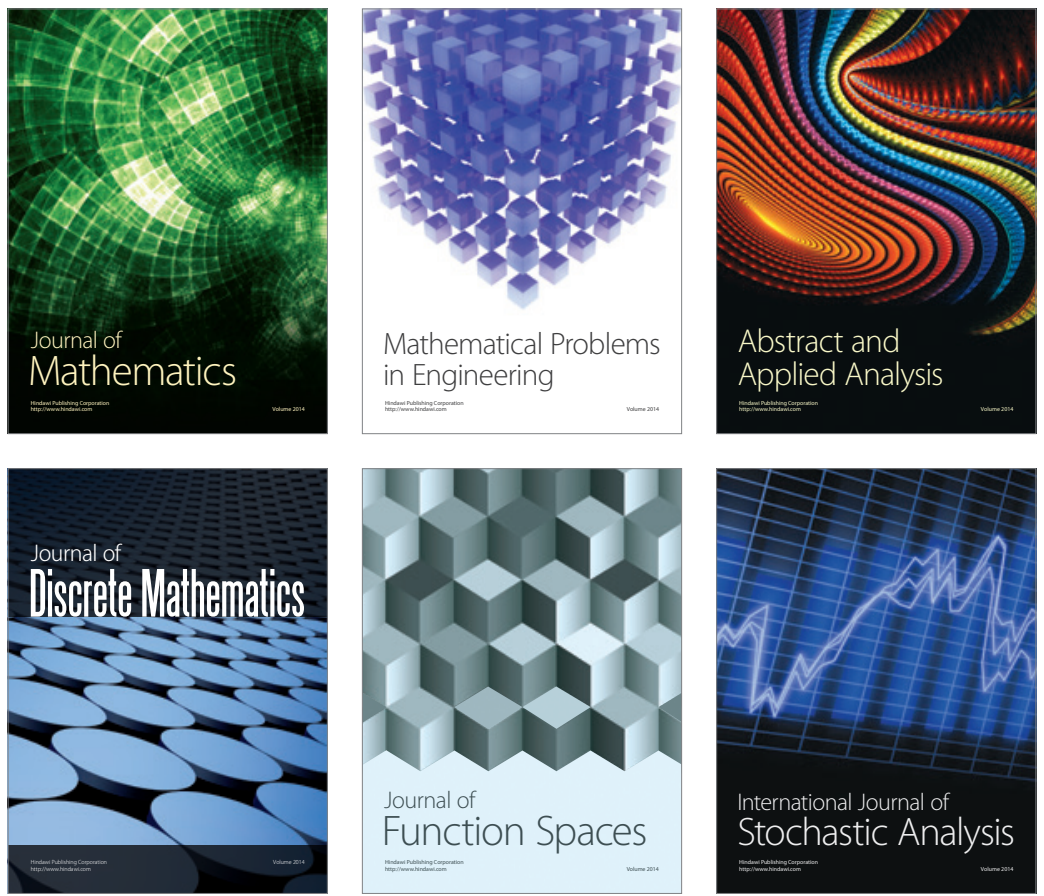

Journal of

Function Spaces

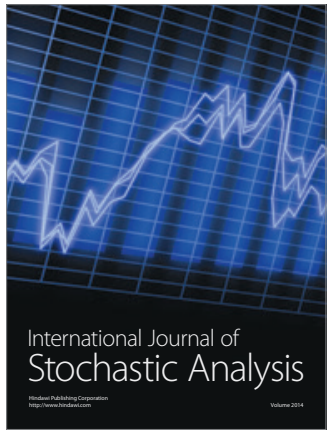

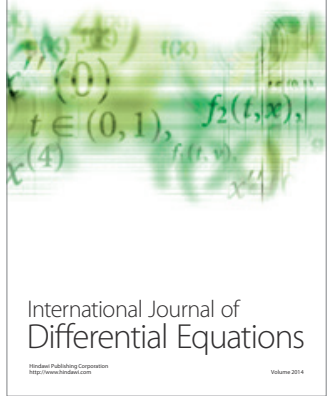
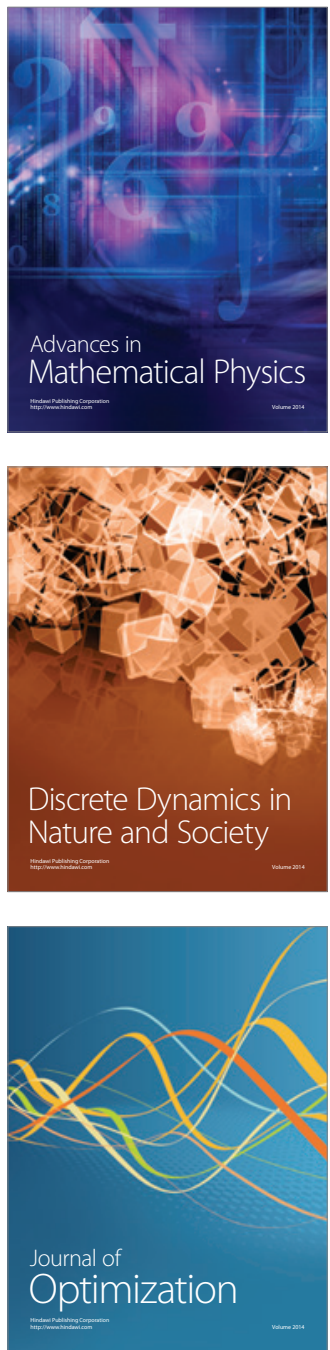